\title{
Disostose espôndilo-costal associada a defeitos de fechamento do tubo neural
}

\author{
Spondylocostal dysostosis associated with neural tube defects
}

Rafael Fabiano M. Rosa1, Paulo Ricardo G. Zen², Rosana Cardoso M. Rosa ${ }^{3}$, Carla Graziadio4, Giorgio Adriano Paskulin ${ }^{5}$

\section{RESUMO}

Objetivo: Salientar a relação dos defeitos de fechamento do tubo neural com a disostose espôndilo-costal (DEC) por meio da descrição de três pacientes.

Descrição dos casos: Paciente 1: menina branca, 22 meses, nascida com mielomeningocele lombar. Na avaliação, apresentava hipotonia, baixa estatura, dolicocefalia, fendas palpebrais oblíquas para cima, pregas epicânticas e tronco curto com tórax assimétrico. A avaliação radiográfica revelou hemivértebras múltiplas, vértebras em borboleta e fusão e ausência de algumas costelas. Paciente 2: menina branca, 22 meses, com moderado atraso do desenvolvimento neuropsicomotor, baixa estatura, olhos profundos, pregas epicânticas, pescoço e tronco curtos com assimetria do tórax, abdome protruso, hemangioma plano na altura da transição lombossacra e fosseta sacral profunda no dorso. A avaliação radiográfica identificou hemivértebras, fusão incompleta de vértebras e vértebras em borboleta, malformações de costelas e espinha bífida oculta em L5/S1. Paciente 3: menina branca, 9 dias de vida, com fendas palpebrais oblíquas para cima, ponte nasal alargada, orelhas baixo implantadas e rotadas posteriormente, tronco curto, tórax assimétrico e meningocele tóraco-lombar. A avaliação radiográfica evidenciou hemivértebras, malformação e ausência de algumas costelas e agenesia diafragmática à esquerda. A tomografia computadorizada de encéfalo mostrou estenose de aqueduto.

Comentários: Vários defeitos de fechamento do tubo neural, de espinha bífida oculta a grandes mielomeningoceles, são observados em pacientes com DEC, indicando que

Instituição: Universidade Federal de Ciências da Saúde de Porto Alegre (UFCSPA) e Complexo Hospitalar Santa Casa de Porto Alegre (CHSCPA), Porto Alegre, RS, Brasil

'Geneticista Clínico da UFCSPA e CHSCPA; Mestre pelo Programa de PósGraduação em Patologia da UFCSPA, Porto Alegre, RS, Brasil

${ }^{2}$ Geneticista Clínico da UFCSPA e CHSCPA; Doutor em Patologia pela UFCSPA; Professor adjunto da Disciplina de Genética Clínica da UFCSPA, Porto Alegre, RS, Brasil

${ }^{3}$ Médica residente do Programa de Residência Médica em Pediatria do Grupo Hospitalar Conceição, Porto Alegre, RS, Brasil

${ }^{4}$ Geneticista Clínica da UFCSPA e CHSCPA; Mestre em Genética e Biologia Molecular pela Universidade Federal do Rio Grande do Sul (UFRGS); Professora assistente da Disciplina de Genética Clínica da UFCSPA, Porto Alegre, RS, Brasil tais pacientes devem ser cuidadosamente avaliados quanto à possível presença desses defeitos.

Palavras-chave: doenças da coluna vertebral; costelas; defeitos do tubo neural; meningocele; mielomeningocele; espinha bífida oculta.

\section{ABSTRACT}

Objective: To highlight the relationship between neural tube defects and spondylocostal dysostosis (SCD) through the description of three patients.

Cases description: Patient 1: white girl, 22 months old, born with a lumbar meningomyelocele. At evaluation, she presented hypotonia, short stature, dolichocephaly, upslanting palpebral fissures, bilateral epicanthal folds, and short trunk with an asymmetric thorax. Radiographic examination showed multiple hemivertebrae, butterfly vertebrae, fusion and absence of some ribs. Patient 2: white girl, 22 months old, with moderate neuropsychomotor delay, short stature, deep set eyes, bilateral epicanthal folds, short neck and trunk with an asymmetric thorax, protruding abdomen, hemangioma at the level of lumbosacral transition and deep sacral dimple. The radiographic evaluation showed hemivertebrae, incomplete fusion of vertebrae and butterfly vertebrae, costal malformations and spina bifida occulta in L5/S1. Patient 3: white girl, nine days old, with upslanting palpebral fissures, broad nasal bridge, anteverted nostrils, low-set and posteriorly rotated ears, short trunk with asymmetric thorax, and thoracolombar meningocele. Radiographic evaluation

${ }^{5}$ Geneticista Clínico da UFCSPA e CHSCPA; Citogenista da UFCSPA; Doutor em Genética e Biologia Molecular pela UFRGS; Professor associado da Disciplina de Genética Clínica da UFCSPA, Porto Alegre, RS, Brasil

Endereço para correspondência:

Giorgio Adriano Paskulin

Rua Sarmento Leite, número 245, sala 403 - Centro

CEP 90050-170 - Porto Alegre/RS

E-mail: paskulin@ufcspa.edu.br

Recebido em: 4/8/08

Aprovado em: 19/10/08 
showed several hemivertebrae, malformation, absence of some ribs, and diaphragmatic agenesia at left. Brain tomography showed an aqueductal stenosis.

Comments: Several neural tube defects, from spina bifida occulta to large meningomyelocele, are observed in patients with SCD. Thus, these patients should be carefully evaluated regarding the possible presence of such defects.

Key-words: spinal diseases; ribs; neural tube defects; meningocele; meningomyelocele; spina bifida occulta.

\section{Introdução}

A disostose espôndilo-costal (OMIM 277300) ${ }^{(1)}$ é uma doença genética rara e etiologicamente heterogênea caracterizada por defeitos variáveis, muitas vezes graves, de costelas e coluna vertebral. A maioria dos casos descritos apresenta um padrão de herança autossômica recessiva associada a mutações no gene DII3 localizado em 19q13 (SCDO1; OMIM 602768), MESP2 situado em 15q26.1 (SCDO2; OMIM 605195) ou LFNG em 7p22 (SCD03; OMIM 602576), importantes componentes da via de sinalização $\operatorname{Notch}^{(1-5)}$. Esta via apresenta relação íntima com um mecanismo de oscilação molecular chamado "relógio da segmentação" (segmentation (lock), implicado no processo de segmentação dos vertebrados, que corresponde a uma repetição, ao longo do eixo anteroposterior, de estruturas similares derivadas das três camadas embrionárias germinativas, sendo que, em humanos, é mais óbvio na coluna vertebral ${ }^{(6)}$. Vale lembrar, entretanto, que uma forma autossômica dominante (OMIM 122600) é relatada com pouca frequência ${ }^{(1,7,8)}$. A associação da disostose espôndilo-costal com defeitos de fechamento do tubo neural é descrita na literatura e muitos autores têm discutido uma possível ligação etiológica entre ambos ${ }^{(5,9-14)}$.

O objetivo deste artigo foi salientar esta relação dos defeitos de fechamento do tubo neural com a disostose espôndilo-costal por meio da descrição dos casos de três pacientes avaliados em nosso Serviço, juntamente com uma revisão da literatura.

\section{Caso 1}

Menina branca de um ano e dez meses de idade, segunda filha de um casal de pais jovens, hígidos e não consanguíneos. A história familiar era negativa para anormalidades congênitas. Nasceu de parto cesáreo com 36 semanas de gestação devido à apresentação pélvica, pesando 2790g (entre P10-
50), medindo $50 \mathrm{~cm}$ (entre P50-90), com perímetro cefálico de $37 \mathrm{~cm}$ (>P90) e Apgar no quinto minuto igual a 9. A criança foi submetida a uma cirurgia para correção de uma mielomeningocele lombossacra no primeiro dia de vida. Ela possuía também hidrocefalia secundária a este defeito.

$\mathrm{Na}$ avaliação clínica, com um ano e dez meses de idade, a criança apresentava peso de $6 \mathrm{~kg}(<\mathrm{P} 3)$, altura de $68,5 \mathrm{~cm}$ $(<\mathrm{P} 3)$, perímetro cefálico de $41 \mathrm{~cm}(<\mathrm{P} 3)$, dolicocefalia, fendas palpebrais oblíquas para cima, pregas epicânticas, tronco curto com assimetria de tórax e cifoescoliose. A avaliação neurológica verificou a presença de hipotonia, paraplegia dos membros inferiores, bexiga neurogênica e importante atraso no desenvolvimento neuropsicomotor: a paciente apresentou sorriso social com cerca de oito meses de idade e, com um ano e dez meses, ainda não possuía sustento cefálico, nem pronunciava dissílabas. A avaliação radiográfica mostrou anormalidades múltiplas de vértebras com presença de fendas vertebrais (regiões radiotransparentes e longitudinais observadas na visão lateral da coluna), hemivértebras, fusão de vértebras e vértebras em borboleta acometendo todos os segmentos da coluna (cervical, torácica e lombar), marcadas anomalias costais do terceiro, quarto e oitavo arcos costais à direita, e do terceiro, quarto e quinto à esquerda, com alargamento, bifidez e fusão de costelas, além de osteopenia difusa (Figura 1A e Tabela 1). A tomografia computadorizada de encéfalo evidenciou uma acentuada dilatação do sistema ventricular, especialmente dos ventrículos laterais. O ultrassom abdominal mostrou dilatação pielocalicial da pelve renal esquerda. O estudo radiográfico das mãos, pés e quadris, a ecocardiografia e o cariótipo de alta resolução ( $\geq 550$ bandas) por bandas GTG foram normais.

\section{Caso 2}

Menina branca de um ano e dez meses de idade, a segunda filha de um casal de pais jovens, hígidos e não consanguíneos. A história familiar era negativa para anormalidades congênitas da coluna. A criança nasceu de parto vaginal pesando $2495 \mathrm{~g}(<\mathrm{P} 3)$, medindo $41 \mathrm{~cm}(<\mathrm{P} 3)$, com perímetro cefálico de $34 \mathrm{~cm}$ (P50) e Apgar no quinto minuto igual a 9. Aos oito meses de idade, foi submetida a uma cirurgia de correção de hérnia inguinal bilateral.

$\mathrm{Na}$ avaliação clínica, com um ano e dez meses, apresentava peso de $9 \mathrm{~kg}(<\mathrm{P} 3)$, altura de $78 \mathrm{~cm}(<\mathrm{P} 3)$, perímetro cefálico de $46 \mathrm{~cm}$ (entre P25-50), cabelos e sobrancelhas esparsas, olhos profundos, pregas epicânticas, pescoço e tórax curtos com assimetria de tórax, cifoescoliose, abdome protruso, 
além de hemangioma plano na linha média da transição lombossacra e fosseta sacral profunda no dorso (Figura 1B e Tabela 1). Mostrava também atraso no desenvolvimento neuropsicomotor: sustentou a cabeça com cerca de seis meses, sentou sem apoio aos dez meses, andou sozinha com um ano e quatro meses e pronunciou as primeiras palavras com um ano e cinco meses. A avaliação da coluna vertebral e do tórax por radiografias e tomografia computadorizada identificou uma pequena costela cervical à direita, sinuosidade moderada da coluna, malformações vertebrais da coluna dorsal e lombar com um grande número de hemivértebras, fusão incompleta de vértebras e vértebras em borboleta, anomalias costais com fusão incompleta da porção posterior de algumas costelas (entre a nona, décima e $11^{\mathrm{a}}$ costela à direita, entre a sexta e a sétima, e a nona e a décima à esquerda), alargamento do arco posterior da nona e décima costelas à direita, e espinha bífida oculta na quinta vértebra lombar (L5) e primeira sacral (S1). A ecocardiografia, o ultrassom abdominal, a radiografia de quadril e o cariótipo de alta resolução ( $\geq 550$ bandas) por bandas GTG foram normais (Tabela 1).

\section{Caso 3}

Menina branca de nove dias de vida, primeira filha de pais hígidos e jovens. Nascida a termo, por parto cesáreo, pesando 3010g (P25), com Apgar igual a 4 e 8 no primeiro e quinto minutos, respectivamente, de uma gestação sem intercorrências.

$\mathrm{Na}$ avaliação clínica, aos nove dias de vida, ela apresentava peso de $2970 \mathrm{~g}$ (P10), perímetro cefálico de $33 \mathrm{~cm}$ (entre P25-50), fendas palpebrais oblíquas para cima, raiz nasal baixa e larga, narinas antevertidas, orelhas baixo implantadas e rodadas posteriormente, tronco curto com tórax assimétrico, escoliose e meningocele tóraco-lombar. A avaliação radiográfica evidenciou uma deformidade de tórax com uma escápula mais elevada à esquerda; escoliose torácica; presença de várias hemivértebras e vértebras fusionadas, especialmente na porção inferior da coluna torácica e em toda lombar; alargamento, fusão e agenesia de costelas, principalmente no segmento inferior do tórax esquerdo; agenesia diafragmática à esquerda e meningocele tóraco-lombar com extensão subcutânea localizada mais à esquerda da linha média. A tomografia computadorizada de encéfalo mostrou hidrocefalia secundária a uma estenose de aqueduto (Figura $1 \mathrm{C}$ e Tabela 1). O ultrassom abdominal, a ecocardiografia e o cariótipo de alta resolução ( $\geq 550$ bandas) por bandas GTG foram normais. A criança evoluiu com instabilidade respiratória e hemodinâmica, falecendo com 17 dias de vida devido à insuficiência cardiorrespiratória.

\section{Discussão}

Desde a descrição, realizada por Jarcho e Levin em $1938^{(15)}$, de dois irmãos com uma condição grave envolvendo malformações vertebrais extensas, diferentes tipos de nanismos de tronco curto caracterizados por múltiplos defeitos de segmentação dos corpos vertebrais têm sido descritos ${ }^{(16)}$. Fazem parte deste grupo, entre outras condições, a síndrome de Klippel-Feil (OMIM 148900), a síndrome de Goldenhar (espectro óculo-aurículo-vertebral) (OMIM 164210), a síndrome de Alagille (OMIM 118450) e a associação VATER/ VACTERL (acrônimo para Vertebral, Anal, Cardiac, Tracheoesophageal, Renal e Limb) (OMIM 192350) $)^{(1,5,6)}$.

A disostose espôndilo-costal, por sua vez, é uma anormalidade rara de segmentação da coluna vertebral caracterizada por alterações vertebrais variáveis que incluem fusão de vértebras, vértebras em borboleta e, especialmente, hemivértebras, além de alterações costais intrínsecas, como alargamento, bifurcação, agenesia e fusão de costelas ${ }^{(2,5,16,17)}$. O envolvimento vertebral é frequentemente extenso, afetando vários segmentos da coluna,

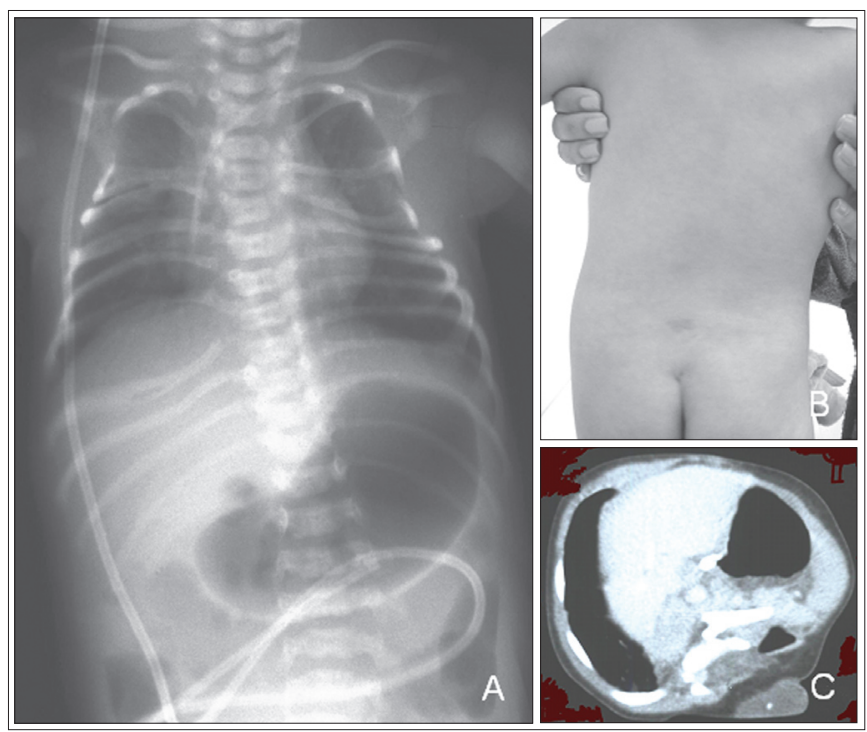

Figura 1 - Radiografia de tórax da Paciente 1 mostrando anormalidades assimétricas de vértebras e costelas $(A)$; visão posterior do tronco da Paciente 2, expondo o tronco curto com a presença de um hemangioma plano na altura da transição tóraco-lombar e uma fosseta sacral profunda (B); tomografia computadorizada de tórax com um corte transversal da Paciente 3 , mostrando uma anormalidade vertebral juntamente com a meningocele torácica $(\mathrm{C})$. 
Tabela 1 - Comparação entre os achados clínicos observados em pacientes com disostose espondilocostal e espondilotorácica (Jarcho Levin) descritos na literatura* com nossos três pacientes

\begin{tabular}{|c|c|c|c|c|c|}
\hline \multirow{2}{*}{ Achados clínicos } & \multirow{2}{*}{ Disostose espôndilo-torácica } & \multirow{2}{*}{ Disostose espôndilo-costal } & \multicolumn{3}{|c|}{ Pacientes } \\
\hline & & & 1 & 2 & 3 \\
\hline Baixa estatura & ++ & + & + & + & + \\
\hline Pescoço curto & + & + & - & + & - \\
\hline Tórax curto & ++ & + & + & + & + \\
\hline Tórax assimétrico & - & + & + & + & + \\
\hline Tórax em leque & + & - & - & - & - \\
\hline Escoliose & + & ++ & + & + & ++ \\
\hline Hemivértebras & + & + & + & + & + \\
\hline Vértebras fusionadas & + & + & + & + & + \\
\hline Vértebras em borboleta & + & + & + & + & - \\
\hline Costelas fusionadas & + (posterior) & + (aleatória) & + & + & + \\
\hline Costelas bífidas & + & + & + & - & - \\
\hline Costelas alargadas & - & + & + & + & + \\
\hline Costelas ausentes & - & + & - & - & + \\
\hline Disfunção cardiorrespiratória & ++ & + & - & - & + \\
\hline Hipotonia & - & + & ++ & + & + \\
\hline Hidrocefalia & - & + & - & - & + \\
\hline Ponte nasal alargada & + & - & + & - & + \\
\hline Orelhas baixo implantadas & + & - & - & - & + \\
\hline Cardiopatia congênita & + & + & - & - & - \\
\hline Abdome protuberante & + & + & + & + & - \\
\hline Hérnia diafragmática & + & + & - & - & + \\
\hline Hérnia inguinal /umbilical & + & + & - & + & - \\
\hline Anomalias renais & + & + & + & - & - \\
\hline Mielomeningocele & - & + & + & - & - \\
\hline Meningocele & - & + & - & - & + \\
\hline Espinha bífida & + & + & - & + & - \\
\hline Ânus imperfurado & + & + & - & - & - \\
\hline
\end{tabular}

*Baseado em Cornier et al $^{(16)}:$ + presente; ++ presente de forma mais intensa.

em especial a região torácica ${ }^{(5)}$. A maioria dos casos relatados apresenta um bom prognóstico devido, em parte, à assimetria das anomalias torácicas, que causa um tórax menos restritivo.

A DSC é frequentemente confundida com outra doença genética chamada disostose espôndilo-torácica (OMIM 277300), também conhecida como síndrome de Jarcho-Levin, uma forma grave de defeito de segmentação da coluna, comumente associada a óbito precoce devido à disfunção ou infecção respiratória. A apresentação clínica da disostose espôndilo-costal difere da observada na disostose espôndilo-torácica por apre- sentar uma forma de nanismo com um encurtamento menos acentuado do tronco associado a anormalidades assimétricas de costelas. Além disso, na disostose espôndilo-costal, observa-se tórax assimétrico sem a configuração em leque (fan-like) ou em caranguejo (crab-like), característico da disostose espôndilotorácica. Na disostose espôndilo-costal, as anormalidades de costelas, como já referido, são também intrínsecas, ao contrário da disostose espôndilo-torácica, na qual as costelas são normais, mas fusionadas posteriormente junto à sua origem vertebral $^{(5,16,18)}$ (Tabela 1$)$. 
Achados adicionais presentes na disostose espôndilo-costal incluem anomalias urogenitais, anorretais e de membros, malformações cerebrais e cardíacas, hérnias diafragmáticas, umbilicais e inguinais, além de defeitos de fechamento do tubo neural ${ }^{(9,10,16)}$. A Tabela 1 sumariza os achados clínicos de nossos pacientes, comparando-os àqueles descritos na disostose espôndilo-costal e espôndilo-torácica.

Defeitos de fechamento do tubo neural de várias formas, variando de espinha bífida oculta a grandes mielomeningoceles, têm sido observados em pacientes com disostose espôndilo-costal ${ }^{(9-12,16)}$. Malformações raras incluem a medula espinhal bipartida tipo $\mathrm{I}^{(12)}$, a lipomielomeningocele ${ }^{(13)} \mathrm{e}$ a mielomeningocele intratorácica ${ }^{(14)}$. Muitos autores acreditam na hipótese de que a associação entre a disostose espôndilo-costal e os defeitos de fechamento do tubo neural possa estar relacionada à interação de diferentes genes, levando a um defeito precoce da gastrulação, que poderia progredir e depois envolver a neurulação primária e secundária ${ }^{(5,11-}$ 13). Entretanto, são necessárias mais evidências para que se confirme a existência dessa ligação etiológica. De qualquer forma, pacientes com disostose espôndilo-costal devem ser cuidadosamente examinados quanto à possível presença de defeitos de fechamento do tubo neural.
Como visto inicialmente, a disostose espôndilo-costal é uma condição genética etiologicamente heterogênea que envolve tanto um padrão de herança autossômico recessivo como dominante ${ }^{(5,16)}$. Os três pacientes relatados eram casos isolados de disostose espôndilo-costal. Mesmo não existindo história de consanguinidade entre os pais, este achado pode ser sugestivo de um possível distúrbio autossômico recessivo (considerado também o padrão de herança mais frequentemente descrito em pacientes com a disostose espôndilo-costal). Contudo, não se pode descartar a possibilidade de que estes pacientes apresentem uma forma autossômica dominante decorrente de uma mutação nova (de novo). Assim, não foi possível definir o tipo de herança específico relacionado à disostose espôndilo-costal, o que também impossibilitou a realização de um adequado aconselhamento genético às famílias dos pacientes. Embora a disostose espôndilo-costal constitua uma doença gênica, a avaliação cariotípica foi realizada com o objetivo de excluir uma possível cromossomopatia, como deleções do braço curto e longo do cromossomo 18 e certas formas de mosaicismo que se associam, algumas vezes, a um fenótipo semelhante, com anormalidades vertebrais e costais ${ }^{(5)}$, o que poderia interferir na forma de avaliação dos pacientes e no cálculo dos riscos de recorrência familiar.

\section{Referências bibliográficas}

1. Online Mendelian Inheritance in Man, OMIM (TM) [homepage on the Internet]. Baltimore e Bethesda: BeMcKusick-Nathans Institute for Genetic Medicine, Johns Hopkins University and National Center for Biotechnology Information, National Library of Medicine [cited 2009 Jul 29]. Available from: http://www. ncbi.nlm.nih.gov/omim/

2. Bulman MP, Kusumi K, Frayling TM, McKeown C, Garrett C, Lander ES et al. Mutations in the human Delta homologue, DLL3, cause axial skeletal defects in spondylocostal dysostosis. Nat Genet 2000;24:438-41.

3. Sparrow DB, Chapman G, Wouters MA, Whittock NV, Ellard S, Fatkin D et al. Mutation of the LUNATIC FRINGE gene in humans causes spondylocostal dysostosis with a severe vertebral phenotype. Am J Hum Genet 2006;78:28-37.

4. Sparrow DB, Chapman G, Turnpenny PD, Dunwoodie SL. Disruption of the somitic molecular clock causes abnormal vertebral segmentation. Birth Defects Res C Embryo Today 2007;81:93-110.

5. Turnpenny PD, Alman B, Cornier AS, Giampietro PF, Offiah A, Tassy O et al. Abnormal vertebral segmentation and the notch signaling pathway. Dev Dyn 2007;236:1456-74

6. Pourquié O, Kusumi K. When body segmentation goes wrong. Clin Genet 2001;60:409-16.

7. Floor E, De Jong RO, Fryns JP, Smulders C, Vles JS. Spondylocostal dysostosis: an example of autosomal dominant transmission in a large family. Clin Genet 1989;36:236-41.

8. Lorenz P, Rupprecht E. Spondylocostal dysostosis: dominant type. Am J Med Genet 1990;35:219-21.

9. Giacoia GP, Say B. Spondylocostal dysplasia and neural tube defects. J Med
Genet 1991;28:51-3.

10. Martínez-Frías ML, Bermejo E, Paisán L, Martín M, Egüés J, López J-A et al. Severe spondylocostal dysostosis associated with other congenital anomalies: a clinical/epidemiologic analysis and description of ten cases from the Spanish Registry. Am J Med Genet 1994;51:203-12.

11. Duru S, Ceylan S, Güvenc BH. Segmental costovertebral malformations: association with neural tube defects. Report of 3 cases and review of the literature. Pediatr Neurosurg 1999;30:272-7.

12. Etus V, Ceylan S, Ceylan S. Association of spondylocostal dysostosis and type I split cord malformation. Neurol Sci 2003;24:134-7.

13. Nadkarni TD, Menon RK, Desai KI, Goel A. Segmental costovertebral malformation associated with lipomyelomeningocoele. J Clin Neurosci 2005;12:599-601.

14. Yi S, Yoon DH, Shin HC, Kim KN, Lee SW. A thoracic myelomeningocele in a patient with spondylocostal dysostosis. Case report. J Neurosurg 2006;104:37-40.

15. Jarcho S, Levin P. Hereditary malformation of the vertebral bodies. Bull John Hopkins Hosp 1938;62:216-26.

16. Cornier AS, Ramirez N, Carlo S, Reiss A. Controversies surrounding JarchoLevin syndrome. Curr Opin Pediatr 2003;15:614-20.

17. Takikawa K, Haga N, Maruyama T, Nakatomi A, Kondoh T, Makita Y et al. Spine and rib abnormalities and stature in spondylocostal dysostosis. Spine 2006;31:E192-7.

18. Karakurt C, Kara C, Akgün D, Sipahi T. Spondylocostal dysostosis: presentation of two cases. Int Pediatr 2001;16:173-5. 